Analele Universităţii de Vest, Timişoara

Seria Matematică - Informatică

LIII, 1, (2015), 37- 56

\title{
Hyperbolic harmonic functions and the associated integral equations
}

Namita Das and Rajendra Prasad Lal

\begin{abstract}
In this paper we consider a class of integral equations associated with the invariant mean value property of hyperbolic harmonic functions. We have shown that nonconstant solutions of these integral equations are functions of unbounded variations and do not attain their supremum or infimum on $[0,1)$. We present an algorithm to obtain numerical solutions of these integral equations. We also consider the equivalent ordinary differential equations and used MATLAB to obtain numerical solutions of these differential equations.
\end{abstract}

AMS Subject Classification (2000). 31B05 ; 31B10 ; 65R20 Keywords. Bergman space, Berezin transform, hyperbolic harmonic functions, invariant mean-value property, integral equations

\section{Introduction}

Ahern, Flores and Rudin [1], Axler, Cuckovic [4] and Yi [9] studied the class of integral equations

$$
g(x)=(1-x)^{n+1} \int_{0}^{1} \frac{n+t x}{(1-t x)^{n+2}} g(t) t^{n-1} d t, n \geq 1
$$

associated with the invariant mean value property of $\mathcal{M}$-harmonic functions. They proved that constants are the only solutions of these integral equations if $n \leq 11$ and this is not true if $n \geq 12$. 
Jevtic [7] studied the equation

$$
g(x)=(1-x)^{\gamma} \frac{\gamma}{2} \int_{0}^{1} \frac{1+t x}{(1-t x)^{\gamma+1}} g(t) t^{\frac{\gamma}{2}-1} d t
$$

$\gamma \geq 2$, that arises naturally in the study of the invariant mean value property of hyperbolically-harmonic functions and showed that if $\gamma \geq 2$, then the constants are the only solutions of the equation (1.2) if and only if $2 \leq \gamma \leq$ $12+\epsilon_{0}$ where $0<\epsilon_{0}<1$. The approach to the problem considered in [7] comes from Yi's work [9].

Let $\mathcal{B}_{n}$ be the open unit ball and $S^{n-1}$ be the unit sphere in $n$-dimensional real Euclidean space $\mathbb{R}^{n}, n \geq 2$. We recall that a similarity is a map $x \rightarrow$ $m x+b$, where $b \in \mathbb{R}^{n}$ and $m=\lambda k$ with $\lambda>0$ and $k \in O(n)$ (as usual, $O(n)$ denotes the group of all orthogonal linear maps on $\mathbb{R}^{n}$ ), and the inversion of $\mathbb{R}^{n} \cup\{\infty\}$ relative to the unit sphere $S^{n-1}$ is defined by $\jmath(x)=x^{*}$ where $x^{*}=\frac{x}{|x|^{2}}$, if $x \neq 0, \infty ; x^{*}=0$ if $x=\infty$ and $x^{*}=\infty$ if $x=0$. For $a, x \in \mathcal{B}_{n}$, define $T_{a}(x)=\left(I-2 Q_{a}\right) G_{a}(x)$ where $G_{a}(x)=a^{*}+\left(\left|a^{*}\right|^{2}-1\right)\left(x-a^{*}\right)^{*}$, and $Q_{a}(x)=<x, a>a^{*}$. Let $\widehat{M}\left(\mathcal{B}_{n}\right)$ be the subgroup of the full Mobius group $\widehat{M}\left(\mathbb{R}^{n}\right)$ generated by all similarities together with the inversion map

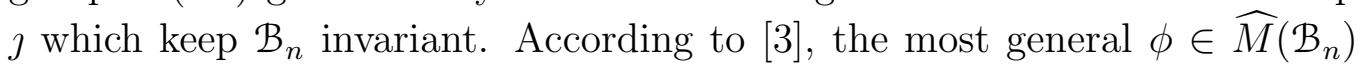
with $\phi(a)=0$ is of the form $k T_{a}$ with $k \in O(n)$.

Definition 1.1. The $C^{2}\left(\mathcal{B}_{n}\right)$ functions on $\mathcal{B}_{n}$ annihilated by the LaplaceBeltrami operator

$$
\triangle_{h}=\left(1-|x|^{2}\right)\left[\left(1-|x|^{2}\right) \triangle+(2 n-4) R\right]
$$

for the Poincare metric on $\mathcal{B}_{n}$ are called hyperbolic-harmonic(h-h) [3]. Here $\triangle$ is the ordinary Laplacian and $R=\sum_{j=1}^{n} x_{j} \frac{\partial}{\partial x_{j}}$.

Note that if $n=2$ (and only then), the classes of harmonic, hyperbolicallyharmonic and $\mathcal{M}$-harmonic functions on $\mathcal{B}_{2}$ coincide. Let $\nu$ denote the Lebesgue measure on $\mathbb{R}^{n}$ normalized so that $\nu\left(\mathcal{B}_{n}\right)=1$ and $\sigma$ denote the normalized surface area measure on $S^{n-1}$.

Suppose $f \in L^{1}\left(\mathcal{B}_{n}, d \nu\right)$ and

$$
\int_{\mathcal{B}_{n}}(f \circ \psi) d \nu=f(\psi(0))
$$

for every $\psi \in \widehat{M}\left(\mathcal{B}_{n}\right)$. The equation (1.4) is called the $\widehat{M}\left(\mathcal{B}_{n}\right)$ invariantvolume mean value property for hyperbolically-harmonic functions, in the 
sense that $f \circ \psi$ has it for every $\psi \in \widehat{M}\left(\mathcal{B}_{n}\right)$, whenever $f$ has it. The fact that $f$ has the invariant volume mean value property is equivalent to saying that

$$
f(x)=\int_{\mathcal{B}_{n}} f\left(T_{-x}(y)\right) d \nu(y)
$$

for $x \in \mathcal{B}_{n}$. Jevtic [7] has shown that if $f \in L^{1}\left(\mathcal{B}_{n}, d \nu\right), n \leq 12$ and

$$
f(x)=\int_{\mathcal{B}_{n}} f\left(T_{-x}(y)\right) d \nu(y)
$$

for $x \in \mathcal{B}_{n}$ then $\triangle_{h} f=0$; this fails for all $n \geq 13$. In [7], it is proved that if $f$ is a radial function and $f(x)=g\left(|x|^{2}\right)$ then (1.5) holds if and only if

$$
g(x)=(1-x)^{n} \frac{n}{2} \int_{0}^{1} \frac{1+t x}{(1-t x)^{n+1}} g(t) t^{\frac{n}{2}-1} d t .
$$

Since the constants are the only radial h-h functions which are integrable and regular at the origin, we conclude that for radial $f \in L^{1}\left(\mathcal{B}_{n}, d \nu\right), f(x)=$ $g\left(|x|^{2}\right)$,

$$
f(x)=\int_{\mathcal{B}_{n}} f\left(T_{-x}(y)\right) d \nu(y)
$$

implies that $f$ is a h-h function if and only if the constants are the only solutions of $(1.6)$ in $L^{1}\left([0,1], t^{\frac{n}{2}-1} d t\right)$. Jevtic [7] showed that this happens if $n \leq 12$ but not if $n \geq 13$.

In fact, he studied the equations

$$
g(x)=(1-x)^{\gamma} \frac{\gamma}{2} \int_{0}^{1} \frac{1+t x}{(1-t x)^{\gamma+1}} g(t) t^{\frac{\gamma}{2}-1} d t
$$

where $\gamma \in \mathbb{R}, \gamma \geq 2$ and established that if $\gamma \geq 2$ then constants are the only solutions of the equation (1.7) if and only if $2 \leq \gamma \leq 12+\epsilon_{0}$ for some $\epsilon_{0} \in(0,1)$. Note that equation (1.7) reduces to equation (1.1) only if $\gamma=2$ and $n=1$.

The integral operator $S_{\gamma}$ associated with the integral equations (1.7) is defined as

$$
\left(S_{\gamma} g\right)(x)=(1-x)^{\gamma} \frac{\gamma}{2} \int_{0}^{1} \frac{1+t x}{(1-t x)^{\gamma+1}} g(t) t^{\frac{\gamma}{2}-1} d t .
$$

In this paper we have shown that nonconstant solutions of (1.2) are functions of unbounded variations and do not attain their supremum or infimum on $[0,1)$. We present an algorithm to obtain numerical solutions of these integral equations. We derive the equivalent ordinary differential equations and used MATLAB to obtain solutions of these differential equations. The 
layout of this paper is as follows: In section 2, we relate Berezin transform with hyperbolic-harmonic functions and prove that if $f \in L^{1}\left(\mathcal{B}_{n}, d \nu\right)$ and $f$ is a fixed point of the Berezin transform $B$ then $f$ is a hyperbolic harmonic function if and only if the constants are the only solutions of the integral equation

$$
g(x)=(1-x)^{n} \frac{n}{2} \int_{0}^{1} \frac{1+x t}{(1-x t)^{n+1}} g(t) t^{\frac{n}{2}-1} d t, n \in \mathbb{N}, n \geq 2
$$

in $L^{1}\left([0,1], t^{\frac{n}{2}-1} d t\right)$.

In section 3 , we obtain the eigenpairs of $S_{\gamma}$ and present some preliminary lemmas describing certain algebraic and intertwining properties of the integral operator $S_{\gamma}$. We then proceed to establish the main result of the paper which states that all nonconstant solutions of the integral equation (1.2) are functions of unbounded variations and present an algorithm to obtain solutions of the integral equation (1.2) for different values of $\gamma \geq 2$. We have shown that the operator $S_{\gamma}$ leaves $L^{\infty}[0,1]$ invariant, is continuous on that space and has spectral radius 1, again on that space. Moreover, any fixed point of $S_{\gamma}$ other than the constants have unbounded variation and do not attain their supremum or infimum on $[0,1)$. In section 4 , we derive the ODE

$$
4 x(1-x)^{2} u^{\prime \prime}+2 n(1-x)^{2} u^{\prime}+2(2 n-4) x(1-x) u^{\prime}=0
$$

with the condition $\int_{0}^{1}|u(t)| t^{\frac{n}{2}-1} d t<\infty$, that is equivalent to the integral equation (1.6). Notice that we are looking for solutions in $L^{1}\left([0,1], t^{\frac{n}{2}-1} d t\right)$, $n \geq 2, n \in \mathbb{N}$. In section 5 , we find those solutions $u$ of (4.2) that satisfy the condition $\int_{0}^{1}|u(t)| t^{\frac{n}{2}-1} d t<\infty$. We use MATLAB to obtain solutions of the ordinary differential equations for different values of $n \in \mathbb{Z}_{+}$.

\section{The Berezin transform}

Suppose $f \in L^{1}\left(\mathcal{B}_{n}, d \nu\right)$. The Berezin transform of $f$ is defined by

$$
B f(x)=\int_{\mathcal{B}_{n}} f\left(T_{-x}(y)\right) d \nu(y)
$$

for $x \in \mathcal{B}_{n}$. The fact that $f$ has the invariant volume mean value property is equivalent to saying that $B f=f$. 
Suppose $f \in L^{1}\left(\mathcal{B}_{n}, d \nu\right)$ and let $B f=f$. A change of variable argument [3] shows that

$$
f(x)=\int_{\mathcal{B}_{n}} f(y) \frac{\left(1-|x|^{2}\right)^{n}}{[x, y]^{2 n}} d \nu(y) .
$$

Here $[x, y]^{2}=1-2 x \cdot y+|x|^{2}|y|^{2}$.

For $u$ a function defined on $\mathcal{B}_{n}$, recall that the radialization of $u$, denoted by $R u$, is the function on $\mathcal{B}_{n}$ defined by

$$
R u(x)=\int_{S^{n-1}} u(|x| \xi) d \sigma(\xi) .
$$

Then, as is well known,

$$
R u(x)=\int_{O(n)} u(L x) d \tau(L),
$$

for all $x \in \mathcal{B}_{n}$. Here $\tau$ is the Haar measure on $O(n)$. From [3] and the invariance of $\tau$ with respect to $O(n)$, we find that $B(R f)=R(B f)$. Therefore, $B f=f$ implies $B(R f)=R f$.

Let $g:[0,1] \rightarrow \mathbb{C}$ be a function such that $R f(x)=g\left(|x|^{2}\right)$. Then we have

$$
g\left(|x|^{2}\right)=\int_{\mathcal{B}_{n}} g\left(|y|^{2}\right) \frac{\left(1-|x|^{2}\right)^{n}}{[x, y]^{2 n}} d \nu(y) .
$$

On simplification, we find that,

$$
g\left(|x|^{2}\right)=n\left(1-|x|^{2}\right)^{n} \int_{0}^{1} \frac{1+|x|^{2} t^{2}}{\left(1-|x|^{2} t^{2}\right)^{n+1}} g\left(t^{2}\right) t^{n-1} d t
$$

or, equivalently,

$$
g(x)=(1-x)^{n} \frac{n}{2} \int_{0}^{1} \frac{1+x t}{(1-x t)^{n+1}} g(t) t^{\frac{n}{2}-1} d t .
$$

It is not so difficult to verify that [7] constants are the only radial hyperbolic harmonic functions which are integrable and regular at the origin. In the following theorem we relate hyperbolic-harmonic functions and invariant mean value property and show that if $f \in L^{1}\left(\mathcal{B}_{n}, d \nu\right)$ and $B f=f$ then $f$ is a hyperbolic harmonic function if and only if the constants are the only solutions of $(2.5)$ in $L^{1}\left([0,1], t^{\frac{n}{2}-1} d t\right)$. Notice that it is sufficient to prove the result only for radial functions in $L^{1}\left(\mathcal{B}_{n}, d \nu\right)$.

Theorem 2.1. Suppose $f \in L^{1}\left(\mathcal{B}_{n}, d \nu\right)$ is radial and assume that constants are the only solutions of (2.5) in $L^{1}\left([0,1], t^{\frac{n}{2}-1} d t\right)$. Then $B f=f$ if and only if $f$ is hyperbolically harmonic. 
Proof. If $f$ is hyperbolically harmonic on $\mathcal{B}_{n}$, the mean value of $f$ over the sphere of radius $0<r<1$ is $f(0)$. If $f$ is also in $L^{1}\left(\mathcal{B}_{n}, d \nu\right)$, it follows from the invariance of $\triangle_{h}$,

$$
\triangle_{h}(f \circ \phi)=\triangle_{h} f \circ \phi, \phi \in \widehat{M}\left(\mathcal{B}_{n}\right)
$$

together with the integration in polar coordinates that

$$
\int_{\mathcal{B}_{n}}(f \circ \psi) d \nu=f(\psi(0))
$$

for every $\psi \in \widehat{M}\left(\mathcal{B}_{n}\right)$.

Thus $f$ satisfies the $\widehat{M}\left(\mathcal{B}_{n}\right)$ invariant volume mean-value-property for hyperbolic harmonic functions. Notice that $f$ has the $\widehat{M}\left(\mathcal{B}_{n}\right)$ invariant volumemean value property is equivalent to saying that $B f=f$.

Now suppose that $B f=f$ and $f$ is radial. Let $f(z)=g\left(|z|^{2}\right)$. Then it follows that

$$
g(x)=(1-x)^{n} \frac{n}{2} \int_{0}^{1} \frac{1+x t}{(1-x t)^{n+1}} g(t) t^{\frac{n}{2}-1} d t .
$$

By our hypothesis $g \equiv c$ (a constant). Hence $f$ is a constant. Therefore, $f$ is hyperbolically harmonic.

\section{On the solutions of the integral equation}

In this section we find the eigenvalues and eigenvectors of the integral operator $S_{\gamma}$ in $L^{1}\left((0,1), x^{\frac{\gamma}{2}-1} d x\right), \gamma \geq 2$. We express the eigenvectors in terms of hypergeometric functions and the eigenvalues in terms of Gamma function. We also relate the integral operator $S_{\gamma}$ with a kind of differential operator $D_{\gamma}$ and discuss certain algebraic and intertwining properties of these operators. We establish that all nonconstant solutions of the integral equation are functions of unbounded variations and present an algorithm to obtain solutions of the integral equation (1.2) for different values of $\gamma \geq 2$.

Let $\Gamma(s)$ stands for the usual Gamma function, which is an analytic function of $s$ in the whole complex plane except for simple poles at the points $\{0,-1,-2, \cdots\}$. In fact

$$
\Gamma(z)=\frac{e^{-\beta z}}{z} \prod_{n=1}^{\infty}\left(1+\frac{z}{n}\right)^{-1} e^{\frac{z}{n}}
$$


where $\beta$ is the Euler's constant; its approximate value is 0.57722 . Hypergeometric series [6] is frequently used in connection with the theory of spherical harmonics. The Pochhammer symbol $(z)_{n}$ is defined by

$$
(z)_{n}=\left\{\begin{array}{l}
\frac{\Gamma(z+n)}{\Gamma(z)}, \text { for } n=0,1,2, \cdots \\
\frac{(-1)^{|n|}}{(1-z)_{|n|}}, \text { for } n=-1,-2, \cdots
\end{array}\right.
$$

The Gauss hypergeometric function $F(\alpha, \beta ; \gamma ; z)$ given by the power series

$$
F(\alpha, \beta ; \gamma ; z)=\sum_{n=0}^{\infty} \frac{(\alpha)_{n}(\beta)_{n}}{(\gamma)_{n} n !} z^{n}
$$

converges absolutely in the open unit disk $\mathbb{D}$ in $\mathbb{C}$ for all complex parameters $\alpha, \beta$ and $\gamma$ with $\gamma \neq 0,-1,-2, \cdots$ and it is a particular solution of the hypergeometric equation

$$
z(1-z) u^{\prime \prime}+[\gamma-(\alpha+\beta+1) z] u^{\prime}-\alpha \beta u=0 .
$$

Suppose $\gamma \in \mathbb{R}, \gamma \geq 2$. Let $d \mu(x)$ be the weighted measure $x^{\frac{\gamma}{2}-1} d x$ on $(0,1)$. Define the integral operator $S_{\gamma}$ as follows:

$$
S_{\gamma} g(x)=(1-x)^{\gamma} \frac{\gamma}{2} \int_{0}^{1} \frac{1+t x}{(1-t x)^{\gamma+1}} g(t) t^{\frac{\gamma}{2}-1} d t .
$$

Since $\frac{\gamma}{2} \frac{1+t x}{(1-t x)^{\gamma+1}}=\frac{\Gamma\left(\frac{\gamma}{2}+1\right)}{\Gamma\left(\frac{\gamma}{2}\right)} F\left(\gamma, \frac{\gamma}{2}+1 ; \frac{\gamma}{2} ; t x\right)$ we have

$$
S_{\gamma} g(x)=\frac{\Gamma\left(\frac{\gamma}{2}+1\right)}{\Gamma\left(\frac{\gamma}{2}\right)}(1-x)^{\gamma} \int_{0}^{1} F\left(\gamma, \frac{\gamma}{2}+1 ; \frac{\gamma}{2} ; t x\right) g(t) t^{\frac{\gamma}{2}-1} d t .
$$

The operator $S_{\gamma}$ is not a bounded operator from $L^{1}((0,1), d \mu(x))$ into $L^{1}((0,1), d \mu(x))$, because $\int_{0}^{1} \frac{(1-x)^{\gamma}(1+t x)}{(1-t x)^{\gamma+1}} x^{\frac{\gamma}{2}-1} d x \rightarrow \infty$ as $t \rightarrow 1$. However, it is a bounded linear operator from $L^{1}((0,1), d \mu(x))$ to $L^{1}((0,1),(1-x) d \mu(x))$. For $\gamma \geq 2$, define

$$
T_{\gamma} f(x)=\left(\frac{\gamma}{2}+1\right) \frac{\gamma}{2}(1-x)^{\gamma+1} \int_{0}^{1} F\left(\gamma+1, \frac{\gamma}{2}+2 ; \frac{\gamma}{2} ; t x\right)(1-t) f(t) t^{\frac{\gamma}{2}-1} d t .
$$

Lemma 3.1. If $f \in L^{1}((0,1), d \mu(x))$ then (i) $T_{\gamma}$ is a bounded linear operator on $L^{1}((0,1), d \mu(x))$ (ii) $S_{\gamma} T_{\gamma} f=T_{\gamma} S_{\gamma} f$. 
Proof. Let $T_{\gamma} f(x)=\int_{0}^{1} K_{\gamma}(x, t) f(t) d \mu(t), \gamma \geq 2$ where

$$
K_{\gamma}(x, t)=\left(\frac{\gamma}{2}+1\right) \frac{\gamma}{2}(1-x)^{\gamma+1} F\left(\gamma+1, \frac{\gamma}{2}+2 ; \frac{\gamma}{2} ; t x\right)(1-t)
$$

To prove (i) we require the following identities (see [5]):

$$
F(a, b, d, z)=\frac{\Gamma(d)}{\Gamma(c) \Gamma(d-c)} \int_{0}^{1} t^{c-1}(1-t)^{d-c-1} F(a, b, c, z t) d t
$$

$\operatorname{Re} d>\operatorname{Re} c>0,|\arg (1-z)|<\pi$

$$
F(a, b, c, z)=(1-z)^{c-a-b} F(c-a, c-b, c, z),|\arg (1-z)|<\pi .
$$

We first show that if $\gamma \geq 2, K_{\gamma}(x, t) \in L^{1}((0,1), d \mu(x))$, for each $0 \leq t \leq 1$ and $\left\|K_{\gamma}(x, t)\right\|_{L^{1}((0,1), d \mu(x))} \leq C$, where $C$ is a constant depending only on $\gamma$. From (3.2) and (3.3) it follows that

$$
\begin{aligned}
& \int_{0}^{1} K_{\gamma}(x, t) d \mu(x) \\
& =\frac{\Gamma\left(\frac{\gamma}{2}+2\right)}{\Gamma\left(\frac{\gamma}{2}\right) \Gamma(2)}(1-t) \int_{0}^{1} x^{\frac{\gamma}{2}-1}(1-x)^{\gamma+1} F\left(\gamma+1, \frac{\gamma}{2}+2 ; \frac{\gamma}{2} ; t x\right) d x \\
& =\frac{\Gamma\left(\frac{\gamma}{2}+2\right) \Gamma(\gamma+2)}{\Gamma(2) \Gamma\left(3 \frac{\gamma}{2}+2\right)} F\left(\frac{\gamma}{2}+1, \gamma, \frac{3 \gamma}{2}+2, t\right) .
\end{aligned}
$$

Since $F\left(\frac{\gamma}{2}+1, \gamma, \frac{3 \gamma}{2}+2, t\right)$ is bounded, the integral $\int_{0}^{1} K_{\gamma}(x, t) d \mu(x)$ is bounded by a constant $C$ where $C$ depends only on $\gamma$. Thus $K_{\gamma}(x, t) \in L^{1}((0,1), d \mu(x))$, $0 \leq t \leq 1, \gamma \geq 2$. Now if $f \in L^{1}((0,1), d \mu(x))$ then

$$
\begin{gathered}
\int_{0}^{1}\left|T_{\gamma} f(x)\right| d \mu(x) \leq\left\|K_{\gamma}(x, t)\right\|_{L^{1}((0,1), d \mu(x))}\|f\|_{L^{1}((0,1), d \mu(x))} \leq \\
C\|f\|_{L^{1}((0,1), d \mu(x))}
\end{gathered}
$$

and the operator $T_{\gamma}$ is a bounded operator on $L^{1}((0,1), d \mu(x))$. To see (ii) notice that $S_{\gamma} T_{\gamma}=T_{\gamma} S_{\gamma}$ if and only if

$$
\Theta(x, u)=(1-u) \int_{0}^{1}(1-t)^{\gamma+1} F\left(\gamma, \frac{\gamma}{2}+1 ; \frac{\gamma}{2} ; t x\right) F\left(\gamma+1, \frac{\gamma}{2}+2 ; \frac{\gamma}{2} ; t u\right) d \mu(t)
$$

is symmetric with respect to $x$ and $u$. This follows by applying Fubini's theorem and multiplying both sides of $S_{\gamma} T_{\gamma}=T_{\gamma} S_{\gamma}$ by $(1-x)^{-\gamma}$. Expanding the two hypergeometric functions $F\left(\gamma, \frac{\gamma}{2}+1 ; \frac{\gamma}{2} ; t x\right)$ and $F\left(\gamma+1, \frac{\gamma}{2}+2 ; \frac{\gamma}{2} ; t u\right)$ 
into power series with coefficients $a_{k}(\gamma)$ and $b_{j}(\gamma)$ and using the beta function, we see that $\Theta(x, u)$ is equal to

$$
(1-u) \sum_{k, j} a_{k}(\gamma) b_{j}(\gamma) \frac{\Gamma\left(\frac{\gamma}{2}+j+k\right) \Gamma(\gamma+2)}{\Gamma\left(3 \frac{\gamma}{2}+2+j+k\right)} x^{k} u^{j}
$$

It is not difficult to see that $b_{j}(\gamma)=\frac{(\gamma+j)\left(\frac{\gamma}{2}+1+j\right)}{\gamma\left(\frac{\gamma}{2}+1\right)} a_{j}(\gamma)$. Using (3.4), we find that $\Theta(x, u)=\frac{\Gamma(\gamma+2)}{\gamma\left(\frac{\gamma}{2}+1\right)} \sum_{k, j} a_{k}(\gamma) a_{j}(\gamma) c_{j, k}(\gamma) x^{k} u^{j}$, where

$$
\begin{aligned}
c_{j, k}(\gamma) & =\frac{\Gamma\left(\frac{\gamma}{2}+j+k-1\right)}{\Gamma\left(3 \frac{\gamma}{2}+1+j+k\right)} \times\left[\frac{(\gamma+j)\left(\frac{\gamma}{2}+1+j\right)\left(\frac{\gamma}{2}+j+k-1\right)}{3 \frac{\gamma}{2}+1+j+k}-j\left(\frac{\gamma}{2}+j-1\right)\right] \\
& =\frac{\Gamma\left(\frac{\gamma}{2}+j+k-1\right)}{\Gamma\left(3 \frac{\gamma}{2}+1+j+k\right)} \frac{(j+k)\left(\frac{\gamma^{2}}{2}+\gamma\right)+(\gamma+2) j k+\gamma\left(\frac{\gamma^{2}}{4}-1\right)}{3 \frac{\gamma}{2}+1+j+k} .
\end{aligned}
$$

Since $c_{j, k}(\gamma)=c_{k, j}(\gamma)$, we have $\Theta(x, u)=\Theta(u, x)$, as claimed.

Lemma 3.2. Let $\lambda \in \mathbb{C}, \lambda=-4 \beta(n-1-\beta), \beta=(n-1) \alpha, \alpha \in \mathbb{C}, n \in \mathbb{N}$ and $f \in C^{2}\left(\mathcal{B}_{n}\right)$. The function $f$ is radial and $\Delta_{h} f=\lambda f$ if and only if $f(z)=c\left(1-|z|^{2}\right)^{\beta} F\left(\beta, \beta-\frac{n}{2}+1 ; \frac{n}{2} ;|z|^{2}\right)$ where $c$ is a constant.

Proof. Consider the function $g_{\alpha}(x)=\int_{S^{n-1}} P_{h}^{\alpha}(x, \zeta) d \sigma(\zeta)$ where $P_{h}(x, \zeta)=$ $\frac{\left(1-|x|^{2}\right)^{n-1}}{[x, \zeta]^{2(n-1)}}, x \in \mathcal{B}_{n}, \zeta \in S^{n-1}$ is the hyperbolically-harmonic Poisson kernel in $\mathcal{B}_{n}$. By [3], the function $g_{\alpha}$ is radial and $\Delta_{h} g_{\alpha}=\lambda g_{\alpha}$. Notice that $(1-z)^{-\lambda}=\sum_{j=0}^{\infty} \frac{\Gamma(\lambda+j)}{\Gamma(\lambda)} \frac{z^{j}}{j !}, z \in \mathcal{B}_{2}, \lambda \in \mathbb{C} \neq 0,-1,-2, \cdots$. The series converges uniformly on each compact subset of $\mathcal{B}_{2}$. By the orthogonality of monomials in $L^{2}(\sigma)$ and by the invariance of $\sigma$, it is not difficult to see that

$$
\begin{aligned}
& \int_{S^{n-1}} \frac{d \sigma(\zeta)}{[x, \zeta]^{2 \beta}}=F\left(\beta, \beta-\frac{n}{2}+1 ; \frac{n}{2} ; x\right) \text {. Thus } \\
& g_{\alpha}(z)=\left(1-|z|^{2}\right)^{\beta} F\left(\beta, \beta-\frac{n}{2}+1 ; \frac{n}{2} ;|z|^{2}\right)=f(z) .
\end{aligned}
$$

It follows from [3] that if $f$ is radial and $\Delta_{h} f=\lambda f$ then

$$
f(z)=c\left(1-|z|^{2}\right)^{\beta} F\left(\beta, \beta-\frac{n}{2}+1 ; \frac{n}{2} ;|z|^{2}\right)
$$

where $c$ is a constant.

Lemma 3.3. Let $\lambda, \beta \in \mathbb{C}, \gamma \in \mathbb{R}, \gamma \geq 2$. Let $\lambda=-4 \beta(\gamma-1-\beta)$. Let

$$
e_{\beta, \gamma}(x)=(1-x)^{\beta} F\left(\beta, \beta-\frac{\gamma}{2}+1 ; \frac{\gamma}{2} ; x\right), x \in(0,1) .
$$

Then $e_{\beta, \gamma} \in L^{1}((0,1), d \mu(x))$ if and only if $-1<R e \beta<\gamma$ and the function $e_{\beta, \gamma}$ is real analytic in $(0,1)$. The function $e_{\beta, \gamma}$ is an eigenvector of $S_{\gamma}$ corresponding to the eigenvalue $\frac{\Gamma(\gamma-\beta) \Gamma(\beta+1)}{\Gamma(\gamma)}$ if $-1<\operatorname{Re} \beta<\gamma$. 
Proof. We shall first show that $e_{\beta, \gamma} \in L^{1}((0,1), d \mu(x))$ if and only if $-1<$ $\operatorname{Re} \beta<\gamma$. Notice that the hypergeometric function satisfies the following (see $[5])$ :

$$
F(a, b, c, z)=(1-z)^{c-a-b} F(c-a, c-b, c, z),|\arg (1-z)|<\pi .
$$

From this it follows that $e_{\beta, \gamma}(x)=(1-x)^{\gamma-\beta-1} F\left(\frac{\gamma}{2}-\beta, \gamma-\beta-1 ; \frac{\gamma}{2} ; x\right)$. If $\operatorname{Re}(c-a-b)>0$, then from [5] it follows that $F(a, b, c, z)$ is bounded. Hence, if $\operatorname{Re} \beta \leq-1$, then since $(1-x)^{\beta} \notin L^{1}((0,1), d \mu(x))$, hence $e_{\beta, \gamma} \notin$ $L^{1}((0,1), d \mu(x))$. Further, if $\operatorname{Re} \beta \geq \gamma$, then since $(1-x)^{\gamma-\beta-1} \notin L^{1}((0,1)$, $d \mu(x))$ hence $e_{\beta, \gamma} \notin L^{1}((0,1), d \mu(x))$. Now it remains to show that if $-1<$ $\operatorname{Re} \beta<\gamma$, then $e_{\beta, \gamma} \in L^{1}((0,1), d \mu(x))$.

If $-1<\operatorname{Re} \beta<\frac{\gamma-1}{2}$, then $F\left(\beta, \beta-\frac{\gamma}{2}+1, \frac{\gamma}{2}, x\right)$ is bounded and therefore $e_{\beta, \gamma} \in L^{1}((0,1), d \mu(x))$. If $\frac{\gamma-1}{2}<\operatorname{Re} \beta<\gamma$, then $(1-x)^{\gamma-\beta-1} \in L^{1}((0,1)$, $d \mu(x))$ and $F\left(\frac{\gamma}{2}-\beta, \gamma-\beta-1, \frac{\gamma}{2}, x\right)$ is bounded. Hence $e_{\beta, \gamma} \in L^{1}((0,1)$, $d \mu(x))$. Now let, $\operatorname{Re} \beta=\frac{\gamma-1}{2}$. If $\gamma=2 \beta+1$ then by [8] it follows that $F\left(\beta, \beta-\frac{\gamma}{2}+1, \frac{\gamma}{2}, x\right) \sim \frac{\Gamma\left(2 \beta-\frac{\gamma}{2}+1\right)}{\Gamma(\beta) \Gamma\left(\beta-\frac{\gamma}{2}+1\right)} \log \frac{1}{1-x}$ as $x \rightarrow 1$. Since $\beta>0, e_{\beta, \gamma} \in$ $L^{1}((0,1), d \mu(x))$. Assume now $\gamma-2 \beta-1 \notin \mathbb{Z}$. From [8], it also follows that

$$
\begin{gathered}
F\left(\beta, \beta-\frac{\gamma}{2}+1, \frac{\gamma}{2}, x\right)= \\
\frac{\Gamma\left(\frac{\gamma}{2}\right) \Gamma(\gamma-2 \beta-1)}{\Gamma\left(\frac{\gamma}{2}-\beta\right) \Gamma(\gamma-\beta-1)} F\left(\beta, \beta-\frac{\gamma}{2}+1,2 \beta-\gamma+2,1-x\right) \\
+\frac{\Gamma\left(\frac{\gamma}{2}\right) \Gamma(2 \beta-\gamma+1)}{\Gamma(\beta) \Gamma\left(\beta-\frac{\gamma}{2}+1\right)}(1-x)^{\gamma-2 \beta-1} F\left(\frac{\gamma}{2}-\beta, \gamma-\beta-1, \gamma-2 \beta, 1-x\right) .
\end{gathered}
$$

Since $(1-x)^{\gamma-\beta-1} \notin L^{1}((0,1), d \mu(x))$, we have $e_{\beta, \gamma} \in L^{1}((0,1), d \mu(x))$.

Now suppose $-1<\operatorname{Re} \beta<\gamma$. We shall prove that

$$
S_{\gamma} e_{\beta, \gamma}(x)=\frac{\Gamma(\gamma-\beta) \Gamma(\beta+1)}{\Gamma(\gamma)} e_{\beta, \gamma}(x) .
$$

We have already checked that $e_{\beta, \gamma} \in L^{1}((0,1), d \mu(x))$ since $-1<\operatorname{Re} \beta<\gamma$. Now $S_{\gamma} e_{\beta, \gamma}(x)=(1-x)^{\gamma}\left(\frac{\gamma}{2} \int_{0}^{1} \frac{t^{\frac{\gamma}{2}-1} e_{\beta, \gamma}(t)}{(1-t x)^{\gamma+1}} d t+\frac{\gamma x}{2} \int_{0}^{1} \frac{t^{\frac{\gamma}{2}} e_{\beta, \gamma}(t)}{(1-t x)^{\gamma+1}} d t\right)$. Let $I_{1}=\int_{0}^{1} \frac{t^{\frac{\gamma}{2}-1} e_{\beta, \gamma}(t)}{(1-t x)^{\gamma+1}} d t$ and $I_{2}=\int_{0}^{1} \frac{t^{\frac{\gamma}{2}} e_{\beta, \gamma}(t)}{(1-t x)^{\gamma+1}} d t$. The following identities are valid (see [5]).

$$
F(a, b, c ; x)=\frac{1}{B(a, c-a)} \int_{0}^{1} \frac{t^{a-1}(1-t)^{c-a-1}}{(1-t x)^{b}} d t, \operatorname{Re} c>\operatorname{Re} a>0
$$


and

$$
F(a, b, c ; 1)=\frac{\Gamma(c-a-b) \Gamma(c)}{\Gamma(c-a) \Gamma(c-b)}, \operatorname{Re}(c-a-b)>0 .
$$

Expanding $F\left(\beta, \beta-\frac{\gamma}{2}+1, \frac{\gamma}{2} ; t\right)$ into a power series, integrating term by term and using (3.5) and (3.3), we find that

$$
\frac{\gamma}{2} I_{1}=(1-x)^{\beta-\gamma} \sum_{m=0}^{\infty} \frac{\Gamma(\beta+m)}{\Gamma(\beta)} \frac{\Gamma\left(\frac{\gamma}{2}+1\right)}{\Gamma\left(\beta-\frac{\gamma}{2}+1\right)} \frac{\Gamma\left(\beta-\frac{\gamma}{2}+1+m\right)}{\Gamma\left(\frac{\gamma}{2}+\beta+1+m\right)} \frac{\Gamma(\beta+1)}{m !} A
$$

where $A=F\left(\beta+1, \beta-\frac{\gamma}{2}+m, \frac{\gamma}{2}+\beta+1+m ; x\right)$.

If $a$ is a negative integer or zero and $j$ is a positive integer, $\frac{\Gamma(a+j)}{\Gamma(a)}$ means $a(a+1) \cdots(a+j-1)$. Now expanding $F\left(\beta+1, \beta-\frac{\gamma}{2}+m, \frac{\gamma}{2}+\beta+1+m ; x\right)$, into a power series, interchanging the order of summation, splitting the sum into two sums and applying (3.6), we get $\frac{1}{2} \gamma I_{1}=S_{1}+S_{2}$, where

$$
\begin{gathered}
S_{1}=(1-x)^{\beta-\gamma} \frac{\Gamma(\gamma-\beta+1) \Gamma(\beta+1)}{\Gamma(\gamma+1)} \sum_{k=0}^{\infty} \frac{\Gamma(\beta+1+k)}{\Gamma(\beta+1)} \frac{\Gamma\left(\beta-\frac{\gamma}{2}+k\right)}{\Gamma\left(\beta-\frac{\gamma}{2}\right)} \\
\frac{\Gamma\left(\frac{\gamma}{2}+1\right)}{\Gamma\left(\frac{\gamma}{2}+1+k\right)} \frac{x^{k}}{k !}
\end{gathered}
$$

and

$$
\begin{gathered}
S_{2}=(1-x)^{\beta-\gamma} \frac{\beta \Gamma(\gamma-\beta) \Gamma(\beta+1)}{\Gamma(\gamma+1)} \sum_{k=0}^{\infty} \frac{\Gamma(\beta+1+k)}{\Gamma(\beta+1)} \\
\frac{\Gamma\left(\beta-\frac{\gamma}{2}+1+k\right)}{\Gamma\left(\beta-\frac{\gamma}{2}+1\right)} \frac{\Gamma\left(\frac{\gamma}{2}+1\right)}{\Gamma\left(\frac{\gamma}{2}+1+k\right)} \frac{x^{k}}{k !} .
\end{gathered}
$$

Repeating the procedure, we see that $\frac{1}{2} x \gamma I_{2}=S_{3}+S_{4}$, where

$$
\begin{gathered}
S_{3}=(1-x)^{\beta-\gamma} \frac{\gamma}{2} \frac{\Gamma(\gamma-\beta+1) \Gamma(\beta+1)}{\Gamma(\gamma+1)} \sum_{k=1}^{\infty} \frac{\Gamma(\beta+k)}{\Gamma(\beta+1)} \frac{\Gamma\left(\beta-\frac{\gamma}{2}+k\right)}{\Gamma\left(\beta-\frac{\gamma}{2}+1\right)} \\
\frac{\Gamma\left(\frac{\gamma}{2}+1\right)}{\Gamma\left(\frac{\gamma}{2}+1+k\right)} \frac{x^{k}}{(k-1) !}
\end{gathered}
$$

and

$$
\begin{gathered}
S_{4}=(1-x)^{\beta-\gamma} \beta \frac{\Gamma(\gamma-\beta) \Gamma(\beta+1)}{\Gamma(\gamma+1)} \sum_{k=1}^{\infty} \frac{\Gamma(\beta+k)}{\Gamma(\beta+1)} \frac{\Gamma\left(\beta-\frac{\gamma}{2}+1+k\right)}{\Gamma\left(\beta-\frac{\gamma}{2}+1\right)} \\
\frac{\Gamma\left(\frac{\gamma}{2}+1\right)}{\Gamma\left(\frac{\gamma}{2}+1+k\right)} \frac{x^{k}}{(k-1) !} .
\end{gathered}
$$

Thus $S_{\gamma} e_{\beta, \gamma}(x)=\frac{\Gamma(\gamma-\beta) \Gamma(\beta+1)}{\Gamma(\gamma)} e_{\beta, \gamma}(x)$. 
Define the differential operator $D_{\gamma}$ as

$$
D_{\gamma} u(x)=4 x(1-x)^{2} \frac{d^{2} u}{d x^{2}}(x)+2 \gamma(1-x)^{2} \frac{d u}{d x}(x)+4(\gamma-2) x(1-x) \frac{d u}{d x}(x) .
$$

If $f \in C^{2}\left(\mathcal{B}_{n}\right)$ is radial, $f(x)=u\left(|x|^{2}\right), \lambda \in \mathbb{C}, \lambda=-4 \beta(n-1-\beta), \beta=$ $(n-1) \alpha, \alpha \in \mathbb{C}, n \in \mathbb{N}$ and $\Delta_{h} f=\lambda f$ then $D_{n} u=\lambda u$.

It is not difficult to check that if $\mu=-4 \beta(\gamma-1-\beta), \mu \in \mathbb{C}$ then $D_{\gamma} e_{\beta, \gamma}=\mu e_{\beta, \gamma}$. Conversely, if $D_{\gamma} e_{\beta, \gamma}=\mu e_{\beta, \gamma}$ then $\mu=-4 \beta(\gamma-1-\beta)$. To see this, let $u=F\left(\beta, \beta-\frac{\gamma}{2}+1 ; \frac{\gamma}{2} ; x\right)$.

Then since $e_{\beta, \gamma}(x)=(1-x)^{\beta} F\left(\beta, \beta-\frac{\gamma}{2}+1 ; \frac{\gamma}{2} ; x\right)$, hence

$$
\begin{gathered}
D_{\gamma} e_{\beta, \gamma}=4(1-x)^{\beta}\left\{( 1 - x ) \left[x(1-x) u^{\prime \prime}+\right.\right. \\
\left.\left.\left(\frac{\gamma}{2}-\left(2 \beta-\frac{\gamma}{2}+2\right) x\right) u^{\prime}\right]+\left[\left(\beta^{2}+\beta-\frac{\beta \gamma}{2}\right) x-\frac{\beta \gamma}{2}\right] u\right\} .
\end{gathered}
$$

Since $F\left(\beta, \beta-\frac{\gamma}{2}+1 ; \frac{\gamma}{2} ; x\right)$ is a solution of

$$
x(1-x) F^{\prime \prime}+\left(\frac{\gamma}{2}-\left(2 \beta-\frac{\gamma}{2}+2\right) x\right) F^{\prime}-\beta\left(\beta-\frac{\gamma}{2}+1\right) F=0,
$$

it follows that $D_{\gamma} e_{\beta, \gamma}=-4 \beta(\gamma-1-\beta) e_{\beta, \gamma}$.

Let $\lambda \in \mathbb{C}$ and $Y_{\lambda}=\left\{f \in C^{2}(0,1): D_{\gamma} f=\lambda f\right.$ and $f$ is bounded near 0$\}$. Henceforth let $\lambda, \beta \in \mathbb{C}, \gamma \in \mathbb{R}, \gamma \geq 2$ and $\lambda=-4 \beta(\gamma-1-\beta)$. Thus $e_{\beta, \gamma} \in Y_{\lambda}$ if $\lambda=-4 \beta(\gamma-1-\beta)$ and if $f \in Y_{0}$ then $f$ is a solution of

$$
u^{\prime \prime}+\frac{\gamma+(\gamma-4) x}{2 x(1-x)} u^{\prime}=0 .
$$

Solving (3.7) directly, one can verify that $Y_{0}$ consists actually of constants.

Lemma 3.4. Let $\lambda=-4 \beta(\gamma-1-\beta), \gamma \geq 2$. Then every $f \in Y_{\lambda}$ is a constant multiple of $e_{\beta, \gamma}$ and is real analytic in $(0,1)$. Further, $e_{\beta, \gamma} \in Y_{\lambda} \cap$ $L^{1}((0,1), d \mu(x))$ if and only if $-1<R e \beta<\gamma$.

Proof. We have already verified that $e_{\beta, \gamma} \in Y_{\lambda}$. Applying the Frobenius method to the second order differential equation $D_{\gamma} f=\lambda f$, we observe that the solution space is two dimensional and when we check the boundedness of its solution near 0 , we see that the solution space is actually one dimensional. Thus if $f \in Y_{\lambda}$ then $f$ is a constant multiple of $e_{\beta, \gamma}$.

$$
\begin{aligned}
& \text { Consider the entire functions } G_{\gamma}(\lambda)=\prod_{j=1}^{\infty}\left(1-\frac{\lambda}{4 j(\gamma-1+j)}\right) \text {. If } \lambda= \\
& -4 \beta(\gamma-1-\beta) \text { then } G_{\gamma}(\lambda)=\frac{\Gamma(\gamma)}{\Gamma(\beta+1) \Gamma(\gamma-\beta)} .
\end{aligned}
$$


For $\lambda=-4 \beta(\gamma-1-\beta)$ with $\beta \in \mathbb{C}, \gamma \geq 2$, we set $\Sigma_{\gamma-1}=\{\beta \in \mathbb{C}$ : $-1<\operatorname{Re} \beta<\gamma\}$ and $\Omega_{\gamma-1}=\left\{\lambda \in \mathbb{C}: \beta \in \Sigma_{\gamma-1}\right\}$. It is easy to see that every $\lambda \in \Omega_{\gamma-1}$ satisfies $\operatorname{Re} \lambda<4 \gamma$.

From Lemma 3.3 and Lemma 3.4 it follows that if $\lambda \in \Omega_{\gamma-1}, \gamma \geq 2$ and $f \in Y_{\lambda} \cap L^{1}((0,1), d \mu(x))$ then $S_{\gamma} f=\frac{f}{G_{\gamma}(\lambda)}$. Thus the operator $S_{\gamma}$ on the space $Y_{\lambda} \cap L^{1}((0,1), d \mu(x))$ has $G_{\gamma}^{-1}(\lambda)$ as its eigenvalue.

For $\gamma \geq 2$, let $E=\left\{f \in L^{1}((0,1), d \mu(x)): S_{\gamma} f=f\right\}=\left\{f \in L^{1}((0,1)\right.$, $\left.d \mu(x)):\left(I-S_{\gamma}\right) f=0\right\}=\operatorname{ker}\left(I-S_{\gamma}\right)$. Thus $E$ is a closed subspace of $L^{1}((0,1), d \mu(x))$ and hence $E$ is a Banach space. Further, if $f \in E$ then $f$ is real analytic as $E \subset$ Range $S_{\gamma}$ and each $f \in E$ is bounded near 0 since $S_{\gamma} f$ is bounded near 0 . If $E=\operatorname{ker}\left(I-S_{\gamma}\right)$ and $f \in E$ then $D_{\gamma} f$ exists as $f$ is real analytic. Let $B_{\gamma}=\left.D_{\gamma}\right|_{E}$, the restriction of $D_{\gamma}$ to $E$.

Lemma 3.5. (i) $B_{\gamma}$ is a bounded linear operator on $E$. (ii) $G_{\gamma} B_{\gamma}=I_{E}$, the identity operator on $E$.

Proof. If $f \in E$ then $B_{\gamma} f=B_{\gamma} S_{\gamma} f=4 \gamma\left(S_{\gamma} f-T_{\gamma} f\right)$. Hence $B_{\gamma} f=4 \gamma(f-$ $\left.T_{\gamma} f\right) \in L^{1}((0,1), d \mu(x))$. Thus $B_{\gamma}$ from $E$ into $L^{1}((0,1), d \mu(x))$ is bounded. Further, since $S_{\gamma} T_{\gamma}=T_{\gamma} S_{\gamma}$, we obtain $S_{\gamma} B_{\gamma} f=4 \gamma\left(S_{\gamma} f-S_{\gamma} T_{\gamma} f\right)=4 \gamma(f-$ $\left.T_{\gamma} S_{\gamma} f\right)=4 \gamma\left(f-T_{\gamma} f\right)=B_{\gamma} f$. Thus $B_{\gamma}$ maps $E$ to $E$. The proof of (ii) follows from [7].

Let $\Omega_{G}=\left\{\lambda \in \Omega_{\gamma-1}: G_{\gamma}(\lambda)=1\right\}$. If $\gamma \geq 2$, then from [7], it follows that $\Omega_{G}$ is finite. The zeros of $G_{\gamma}$ are at $4 \gamma, 8(\gamma+1), \cdots$, hence none is in $\Omega_{\gamma-1}$. Further $0 \in \Omega_{G}$ for all $\gamma \geq 2$ and that $\Omega_{G}$ contains no other real numbers. This is so since $G_{\gamma}$ is real on the real axis and logarithmic differentiation shows that $G_{\gamma}^{\prime}(\lambda)=G_{\gamma}(\lambda) \sum_{j=1}^{\infty} \frac{1}{\lambda-4 j(\gamma-1+j)} \neq 0$ as $G_{\gamma}(\lambda) \neq 0$ for $\lambda \in$ $\Omega_{\gamma-1}$ and each of these summands has negative real part when $\operatorname{Re} \lambda<4 \gamma$ and hence for all $\lambda \in \Omega_{\gamma-1}$.

Lemma 3.6. For $\gamma \geq 2, \Omega_{G}=\sigma_{p}\left(B_{\gamma}\right)$, the point spectrum of $B_{\gamma}$.

Proof. Suppose $B_{\gamma} f=\lambda f$ for some nonzero $f \in E$. Then by Lemma 3.4, $f \in Y_{\lambda} \cap L^{1}((0,1), d \mu(x))$ and hence $\beta \in \Sigma_{\gamma-1}$. That is, $\lambda \in \Omega_{\gamma-1}$. Since $S_{\gamma} f=f$, we obtain $G_{\gamma}(\lambda)=1$. So $\lambda \in \Omega_{G}$. Conversely, assume $\lambda \in \Omega_{G} \subset$ $\Omega_{\gamma-1}$. Then $e_{\beta, \gamma} \in Y_{\lambda} \cap L^{1}((0,1), d \mu(x)) \neq\{0\}$ and we have $S_{\gamma} f=f$ for all $f \in Y_{\lambda} \cap L^{1}((0,1), d \mu(x))$. Thus $E \supset Y_{\lambda} \cap L^{1}((0,1), d \mu(x))$ and hence $B_{\gamma} e_{\beta, \gamma}=\lambda e_{\beta, \gamma}$ with $e_{\beta, \gamma} \in E$.

For $\gamma \geq 1,-1<\operatorname{Re} \beta<\gamma+1$, let $\Phi_{\gamma}(\beta)=\frac{\Gamma(\beta+1) \Gamma(\gamma-\beta+1)}{\Gamma(\gamma+1)}$. The function $\Phi_{\gamma}$ has simple poles at the negative integers and at $\gamma+1, \gamma+2, \gamma+3, \cdots$. It 
takes real values on the rest of the real axis and nowhere take the value 0 . For $\gamma \geq 2$ and $-1<\operatorname{Re} \beta<\gamma$, let $\Psi_{\gamma}(\beta)=\frac{\Gamma(\gamma-\beta) \Gamma(\beta+1)}{\Gamma(\gamma)}$. Then $\Psi_{\gamma}(\beta)=\Phi_{\gamma-1}(\beta)$. We have shown that $\Psi_{\gamma}(\beta)$ are the eigenvalues of $S_{\gamma}$.

In the following theorem, we show that $S_{\gamma}$ leaves $L^{\infty}[0,1]$ invariant, is continuous on that space and has spectral radius 1, again on that space. Moreover we shall show that any fixed points of $S_{\gamma}$ other than the constants have unbounded variation and do not attain their supremum or infimum on $[0,1)$.

Theorem 3.7. The operator $S_{\gamma}$ is a bounded operator from $L^{\infty}[0,1]$ into $L^{\infty}[0,1]$ and has spectral radius 1 . Moreover, any fixed points of $S_{\gamma}$ other than the constants in $C[0,1) \cap L^{1}[0,1]$ have unbounded variation and do not attain their supremum or infimum on $[0,1)$.

Proof. The points to note are:

(a) Constant functions are fixed points.

(b) If $u \geq 0$ on $[0,1), u \in C([0,1))$ and $u$ is not identically zero then $S_{\gamma} u>0$ on $[0,1)$.

These statements can be verified as follows: Recall that

$$
(1-z)^{-(\gamma+s)}=\sum_{k=0}^{\infty} \frac{\Gamma(\gamma+s+k)}{\Gamma(\gamma+s)} \frac{x^{k}}{k !}, x \in \mathcal{B}_{2} .
$$

From the identity (see [5])

$$
F(a, b, d ; z)=\frac{\Gamma(d)}{\Gamma(c) \Gamma(d-c)} \int_{0}^{1} t^{c-1}(1-t)^{d-c-1} F(a, b, c ; z t) d t
$$

$\operatorname{Re} d>\operatorname{Re} c>0,|\arg (1-z)|<\pi$, it follows that

$$
(1-x)^{\gamma} \frac{\gamma}{2} \frac{1+t x}{(1-t x)^{\gamma+1}}=(1-x)^{\gamma} \frac{\gamma}{2} F\left(\gamma, \frac{\gamma}{2}+1 ; \frac{\gamma}{2} ; t x\right) \in L^{1}((0,1), d \mu(t))
$$

By expanding the hypergeometric function into a power series and applying the beta function together with the negative binomial series, it is also not difficult to see that $(1-x)^{\gamma} \frac{\gamma}{2} \int_{0}^{1} \frac{1+t x}{(1-t x)^{\gamma+1}} t^{\frac{\gamma}{2}-1} d t=1$ for each $\gamma \geq 2$. This proves (a) and (b) since the kernel is positive.

Thus it follows that if $u \in L^{\infty}$ then $S_{\gamma} u \in L^{\infty}$ and $\left\|S_{\gamma} u\right\|_{\infty} \leq\|u\|_{\infty}$. Therefore $\left\|S_{\gamma}\right\| \leq 1$. When $u$ is a constant function, we have $\left\|S_{\gamma} u\right\|_{\infty}=\|u\|_{\infty}$. Hence $\left\|S_{\gamma}\right\|=1$ and the spectral radius of $S_{\gamma}$ is 1 . From these, it also follows that if $u$ is a nonconstant fixed point in $C([0,1))$ then

(i) $\inf _{s \in[0,1)} u(s)<u(t)$ for all $t \in[0,1)$. 
(ii) $\sup _{s \in[0,1)} u(s)>u(t)$ for all $t \in[0,1)$. $s \in[0,1)$

(iii) $\liminf _{t \rightarrow 1} u(t)=\inf _{s \in[0,1)} u(s)$.

(iv) $\limsup _{t \rightarrow 1} u(t)=\sup _{s \in[0,1)} u(s)$.

If $u$ is unbounded below, then (i) and (iii) are trivial. If $u$ is bounded below, let $\alpha=\inf _{t \in[0,1)} u(t)$. Now, $u(t)-\alpha \geq 0$ and is not identically zero since $u$ is not constant. By (a) and (b), $u-\alpha=S_{\gamma}(u-\alpha)>0$ on $[0,1)$, proving (i). Again (iii) is now immediate by continuity. If $u$ is unbounded above, then (ii) and (iv) are trivial. If $u$ is bounded above, the same argument as above applied to $\sup u(t)-u$ shows (ii) and (iv). What (i)-(iv) show $t \in[0,1)$

is that nonconstant $C([0,1))$ fixed points of $S_{\gamma}$ are functions of unbounded varations where either the infimum or supremum may be infinite. They oscillate infinitely many times, thus having unbounded variation on $[0,1)$, not attaining their supremum or infimum anywhere, but approaching both at 1 .

We next give an algorithm for computing the fixed points of the integral operator $S_{\gamma}$ associated with the invariant-mean-value property of hyperbolicharmonic functions. In Algorithm 1, we are actually truncating the infinite series of $F(z, z-\gamma / 2+1, \gamma / 2, x)$ to $\mathrm{L}$ terms. Higher the value of $\mathrm{L}$ higher will be the accuracy of computation of $F(z, z-\gamma / 2+1, \gamma / 2, x)$.

From Lemma 3.3 and Lemma 3.4 it follows that in Algorithm 1, if $\lambda=$ $-4 z(\gamma-1-z)$ then $G_{\gamma}(\lambda)=\prod_{j=1}^{\infty}\left(1-\frac{\lambda}{4 j(\gamma-1+j)}\right)=1$ and $S_{\gamma} f=f$.

If $2 \leq \gamma \leq 12$, in Algorithm 1 then from [7], it follows that $z=0$ or $\gamma-1$. In that case, $f \equiv c$ as $F\left(\gamma-1, \frac{\gamma}{2}, \frac{\gamma}{2}, x\right)=(1-x)^{1-\gamma}$.

The formulation in if-else part used in Algorithm 1 for $\gamma \in \mathbb{N}$ can be easily solved numerically. We illustrate the formulation for $\gamma=3$. Taking $\gamma=3$ and substituting in $\Psi_{\gamma}(z)-1=0$ we get

$$
\Gamma(z+1) \Gamma(3-z)=\Gamma(3) .
$$

Using, Euler's reflection formula, for $z \in \mathbb{C} \backslash \mathbb{Z}$

$$
\Gamma(z) \Gamma(1-z)=\frac{\pi}{\sin (\pi z)}
$$

and replacing $z$ by $z-2$ we get

$$
\Gamma(z-2) \Gamma(3-z)=\frac{\pi}{\sin (\pi(z-2))} .
$$


$\overline{\text { Algorithm } 1 \text { Solutions of integral equations associated with hyperbolic- }}$ harmonic functions

Require: $\gamma(\geq 2) \in \mathbb{R},\left(S_{\gamma} g\right)(x)=(1-x)^{\gamma} \frac{\gamma}{2} \int_{0}^{1} \frac{1+t x}{(1-t x)^{\gamma+1}} g(t) t^{\frac{\gamma}{2}-1} d t, L \in$ $\mathbb{N}$.

Ensure: $f$ is a fixed point of $S_{\gamma}$.

Compute $\Psi_{\gamma}(\beta)=\frac{\Gamma(\beta+1) \Gamma(\gamma-\beta)}{\Gamma(\gamma)}, \quad-1<\operatorname{Re} \beta<\gamma$.

if $\gamma \in \mathbb{N}$ then

if $z \in \mathbb{Z}$ then

The solutions of $\Psi_{\gamma}(z)=1$ are $z=0$ and $\gamma-1$ only else

solve for $z \in \mathbb{C}$ such that $\pi z \prod_{i=1}^{\gamma-1}(z-i)=(-1)^{\gamma-1} \Gamma(\gamma) \sin (\pi z)$

\section{end if}

else

solve for $z \in \mathbb{C}$ such that $\Psi_{\gamma}(z)=1$

\section{end if}

$F(z, z-\gamma / 2+1, \gamma / 2, x)=0$

for $\mathrm{k}=0$ to $\mathrm{L}$ do

$$
F(z, z-\gamma / 2+1, \gamma / 2, x)=F(z, z-\gamma / 2+1, \gamma / 2, x)+\frac{\frac{\Gamma(z+k)}{\Gamma(z)} \frac{\Gamma(z-\gamma / 2+1+k)}{\Gamma(z-\gamma / 2+1)}}{\frac{\Gamma(\gamma / 2+k)}{\Gamma(\gamma / 2)}} \frac{x^{k}}{k !}
$$

end for

Find $f(x)=c(1-x)^{z} F\left(z, z-\frac{\gamma}{2}+1, \frac{\gamma}{2}, x\right), x \in[0,1)$ where $c$ is an arbitrary constant. 
Also $\Gamma(1+z)=z(z-1)(z-2) \Gamma(z-2)$. Substituting in (3.8) we get,

$$
\pi z(z-1)(z-2)=\Gamma(3) \sin (\pi z)
$$

Equating real and imaginary parts we obtain the system of equations

$$
\begin{gathered}
\pi\left(x^{3}-3 x y^{2}+3 y^{2}-3 x^{2}+2 x\right)-\cosh (\pi y) \sin (\pi x)=0 \\
\pi\left(3 x^{2} y-6 x y-y^{3}+2 y\right)-\sinh (\pi y) \cos (\pi x)=0 .
\end{gathered}
$$

The above system can be solved by using generalized Newton-Raphson method [2]. The solutions are listed in Table 1. Also $\pi z(z-1)(z-2)=\Gamma(3) \sin (\pi z)$ can be solved directly in terms of $z$ using symbolic computation tools of MATLAB/MAPLE. Here one may observe that for $n=3$ the solutions $z$ that satisfy the condition $-1<\operatorname{Re} z<n+1$ are only 0 and 2 .

Table 1: Values of $z$ for different $n$

\begin{tabular}{ccc}
\hline \hline $\mathrm{n}=3$ & $\mathrm{n}=12$ & $\mathrm{n}=13$ \\
\hline$-0.0016512+0.00164732 \mathrm{i}$ & $1.000000+0.000001 \mathrm{i}$ & $11.99998+0.00002 \mathrm{i}$ \\
$-0.0015396+0.0015362 \mathrm{i}$ & 0.0000000 & $12.94279+1.86598 \mathrm{i}$ \\
$0.0023145+0.00023068 \mathrm{i}$ & 1.0000000 & $12.94277+1.85432 \mathrm{i}$ \\
$2.00172405+0.001719 \mathrm{i}$ & $10.999996+0.000030 \mathrm{i}$ & 11.999996543 \\
$2.00161288+0.0001609 \mathrm{i}$ & $12.0024538+1.87947 \mathrm{i}$ & 12.000001 \\
$4.00489658+1.3926428 \mathrm{i}$ & $10.9999714-0.0000103 \mathrm{i}$ & $12.000005+0.000002 \mathrm{i}$ \\
$6.12710495+1.8643223 \mathrm{i}$ & $11.000082+0.0000032 \mathrm{i}$ & 0.00000000 \\
$0.0001615+0.00013472 \mathrm{i}$ & $11.000066+0.0000003 \mathrm{i}$ & $12.0000145+0.000001 \mathrm{i}$ \\
\hline
\end{tabular}

The solutions obtained are in conformation with the theoretical results obtained in the lemmas of section 3 (see Lemma 3.3 ). We also observe that nonconstant solutions of the integral equation (1.2) are functions of unbounded variations and are expressed through hypergeometric functions.

\section{Derivation of the differential equation associated with the invariant MVP of hyperbolic harmonic functions}

In this section, we derive the ODE

$$
4 x(1-x)^{2} u^{\prime \prime}+2 n(1-x)^{2} u^{\prime}+2(2 n-4) x(1-x) u^{\prime}=0
$$

with the condition $\int_{0}^{1}|u(t)| t^{\frac{n}{2}-1} d t<\infty$, that is equivalent to the integral equation (1.6). Notice that we are looking for solutions in $L^{1}\left([0,1], t^{\frac{n}{2}-1} d t\right)$, $n \geq 2, n \in \mathbb{N}$. 
Theorem 4.1. Suppose the radial function $f \in C^{2}\left(\mathcal{B}_{n}\right)$ has the form $f(x)=$ $u\left(|x|^{2}\right)=u(t)$. Then $f$ is hyperbolically-harmonic if and only if

$$
4 x(1-x)^{2} u^{\prime \prime}+2 n(1-x)^{2} u^{\prime}+2(2 n-4) x(1-x) u^{\prime}=0 .
$$

Proof. Suppose $f$ is hyperbolically-harmonic. Then $\triangle_{h} f=0$, that is,

$$
\left(1-|x|^{2}\right)^{2} \triangle f+\left(1-|x|^{2}\right)(2 n-4) \sum_{j=1}^{n} x_{j} \frac{\partial f}{\partial x_{j}}=0 .
$$

Now

$$
\left(1-|x|^{2}\right)^{2} \triangle f=\left(1-|x|^{2}\right)^{2} \sum_{j=1}^{n} \frac{\partial^{2} u}{\partial x_{j}^{2}} .
$$

Since $\frac{\partial u}{\partial x_{j}}=\frac{\partial u}{\partial t} \frac{\partial t}{\partial x_{j}}=u^{\prime} 2 x_{j}$, hence $\frac{\partial^{2} u}{\partial x_{j}^{2}}=2\left[u^{\prime \prime} 2 x_{j}^{2}+u^{\prime}\right]$. Thus

$$
\begin{aligned}
\left(1-|x|^{2}\right)^{2} \triangle f & =2\left(1-|x|^{2}\right)^{2} \sum_{j=1}^{n} 2 u^{\prime \prime} x_{j}^{2}+2\left(1-|x|^{2}\right)^{2} \sum_{j=1}^{n} u^{\prime} \\
& =4\left(1-|x|^{2}\right)^{2}|x|^{2} u^{\prime \prime}+2 n\left(1-|x|^{2}\right)^{2} u^{\prime} .
\end{aligned}
$$

Further,

$$
\sum_{j=1}^{n} x_{j} \frac{\partial u}{\partial x_{j}}=\sum_{j=1}^{n} x_{j}\left[\frac{\partial u}{\partial t} \frac{\partial t}{\partial x_{j}}\right]=\sum_{j=1}^{n} x_{j}\left[u^{\prime} 2 x_{j}\right]=2 u^{\prime} \sum_{j=1}^{n} x_{j}^{2}=2 u^{\prime}|x|^{2} .
$$

It follows therefore that $\triangle_{h} f=0$ if and only if

$$
4|x|^{2}\left(1-|x|^{2}\right)^{2} u^{\prime \prime}+2 n\left(1-|x|^{2}\right)^{2} u^{\prime}+\left(1-|x|^{2}\right)(2 n-4) 2 u^{\prime}|x|^{2}=0 .
$$

That is, if and only if

$$
4 x(1-x)^{2} u^{\prime \prime}+2 n(1-x)^{2} u^{\prime}+2(2 n-4) x(1-x) u^{\prime}=0 .
$$

\section{Solutions of the ordinary differential equations}

In this section we find those solutions $u$ of (4.2) that satisfy the condition $\int_{0}^{1}|u(t)| t^{\frac{n}{2}-1} d t<\infty$. We use MATLAB to obtain solutions of the ordinary 
differential equations for different values of $n \in \mathbb{Z}_{+}$. The equation (4.2) can be solved by converting it to an exact form. We rewrite (4.2) as

$$
2 x(1-x) u^{\prime \prime}=(4 x-n x-n) u^{\prime} .
$$

Rearranging the terms in (5.1) we obtain

$$
\frac{u^{\prime \prime}}{u^{\prime}}=\frac{(4-n) x-n}{2 x(1-x)} .
$$

Integrating (5.2) we get,

$$
\ln \left(u^{\prime}\right)=\ln \left(B(1-x)^{n-2} x^{-n / 2}\right)
$$

where $B$ is a constant. Taking antiderivatives of the functions in equation (5.3) we obtain the solution $u(x)$ given by

$$
u(x)=\int_{0}^{x} B(1-t)^{n-2} t^{-n / 2} d t .
$$

For different values of $n \in \mathbb{Z}_{+}$, the solution $u(x)$ can be obtained explicitly by evaluating the indefinite integral in (5.4). We have verified that the constants are the only solutions of (4.2) if $n \leq 12$. The solutions (particular) $u(x)$ for different values of $n$ can be plotted in MATLAB.

1. For $\mathrm{n}=1$ we have $u(x)=A+B(\log (-2-2 \sqrt{x})-\log (2 \sqrt{x}-2))$ and the integral $\int_{0}^{1}|u(x)| x^{\frac{n}{2}-1} d x<\infty$ but $u$ is not a twice continuously differentiable function on $[0,1)$ unless $B=0$. Hence for $n=1$ the solutions $u$ of (4.2) that are in $C^{2}\left(\mathcal{B}_{n}\right)$ and satisfy $\int_{0}^{1}|u(t)| t^{\frac{n}{2}-1} d t<\infty$ are only constant functions.

2. For $\mathrm{n}=2$ we have $u(x)=A+B \log (x)$.

3. For $\mathrm{n}=3$ we have $u(x)=A+B \frac{-2 x-2}{\sqrt{x}}$.

4. For $\mathrm{n}=11$ we have $u(x)=-\left(315 x^{9 / 2}\right)^{-1}\left((2 x+2)\left(35 x^{8}-440 x^{7}+2708 x^{6}-\right.\right.$ $\left.\left.11528 x^{5}+51218 x^{4}-11528 x^{3}+2708 x^{2}-440 x+35\right)\right)$.

5. For $\mathrm{n}=12$ we obtain $u(x)=\left(x^{-5}\right)\left(1 / 5 x^{10}-5 / 2 x^{9}+15 x^{8}-60 x^{7}+210 x^{6}-\right.$ $\left.252 x^{5} \log (x)-210 x^{4}+60 x^{3}-15 x^{2}+5 / 2 x-1 / 5\right)$.

Thus we verify that for $n \in \mathbb{N}, n \leq 12$, the only solutions $u$ of (4.2) that are twice continuously differentiable on $[0,1)$ and satisfy the condition $\int_{0}^{1}|u(t)| t^{\frac{n}{2}-1} d t<\infty$ are constants. This is in conformation to the theoretical result obtained in lemmas of section 3 . 


\section{References}

[1] Ahern P. Flores M. and Rudin W., An invariant volume-mean-value property, J.Funct. Anal., 111, (1993), 380-397.

[2] Atkinson K.E., An Introduction to Numerical Analysis, John Wiley, New York, 1988.

[3] Ahlfors L., Mobius transformations in several dimensions, University of Minnesota, 1981.

[4] Axler S. and Cuckovic Z., Commuting Toeplitz operators with harmonic symbols, Integral Equations Operator Theory, 14, (1991), 1-12.

[5] Erdelyi A. Magnus W. Oberhettinger F. and Tricomi F.G., Higher Transcendental Functions, McGraw-Hill, New York, 1955.

[6] Gelfand I. M., Spherical functions on symmetric Riemannian spaces, Dokl. Akad. Nauk SSSR, 70, (1950), 5-8.

[7] Jevtic M., Fixed points of an integral operator, J. Anal. Math., 91, (2003), 123-141.

[8] Whittaker E. and Watson G., A course of Modern Analysis, Cambridge University Press, 1988.

[9] Yi J.S., A characterization of the functions fixed by a class of integral operators, 1995.

Namita Das

P.G. Department of Mathematics

Utkal University

Vanivihar, Bhubaneswar, 751004,

Odisha, India

E-mail: namitadas440@yahoo.co.in

Rajendra Prasad Lal

Department of Computer and Information Sciences

University of Hyderabad

Hyderabad, India-500046

E-mail: rlal77@gmail.com

Received: 09.09.2014

Accepted: 05.07.2015

Revised: 27.06.2015 